\title{
Quantum correction to chaos in Schwarzian theory
}

\author{
Yong-Hui Qi, ${ }^{a}$ Sang-Jin $\operatorname{Sin}^{a}$ and Junggi Yoon $^{b}$ \\ ${ }^{a}$ Department of Physics, Hanyang University, \\ Seoul, 04763, Korea \\ ${ }^{b}$ School of Physics, Korea Institute for Advanced Study \\ 85 Hoegiro Dongdaemun-gu, Seoul 02455, Republic of Korea \\ E-mail: yhqi@pku.edu.cn, sjsin@hanyang.ac.kr, junggiyoon@kias.re.kr
}

Abstract: We discuss the quantum correction to chaos in the Schwarzian theory. We carry out the semi-classical analysis of the Schwarzian theory to study Feynman diagrams of the Schwarzian soft mode. We evaluate the contribution of the soft mode to the out-oftime-order correlator up to order $\mathcal{O}\left(g^{4}\right)$. We show that the quantum correction of order $\mathcal{O}\left(g^{4}\right)$ by the soft mode decreases the maximum Lyapunov exponent $\frac{2 \pi}{\beta}$.

Keywords: AdS-CFT Correspondence, Effective Field Theories, Field Theories in Lower Dimensions, 2D Gravity

ArXIV EPRINT: 1906.00996 


\section{Contents}

1 Introduction 1

2 Schwarzian theory 2

2.1 Review 2

2.2 Soft mode propagator 4

3 Out-of-time-ordered correlator $\quad 6$

$\begin{array}{ll}3.1 & \text { Dressed bi-local field }\end{array}$

3.2 Euclidean four point function 8

$\begin{array}{lll}3.3 & \text { Real-time out-of-time-ordered correlator } & 12\end{array}$

4 Conclusion $\quad 15$

\section{Introduction}

Recently, quantum chaos has been intensively investigated in AdS/CFT. One of the diagnosis of the chaos is the well-known butterfly effect which stands for the sensitivity of a system on the initial condition. In quantum system, such sensitivity can be captured by the out-of-time-ordered correlator (OTOC) defined by [1-5]

$$
\langle V(t) W(0) V(t) W(0)\rangle_{\beta},
$$

where $\langle\cdots\rangle_{\beta}$ denotes the thermal expectation value at temperature $\beta^{-1}$. The sensitivity of a chaotic system on the initial condition leads to an exponential growth of the OTOC between the dissipation time $t_{d} \sim \beta$ and the scrambling time $t_{*}[1-5]$ :

$$
\frac{\langle V(t) W(0) V(t) W(0)\rangle_{\beta}}{\langle V(t) V(t)\rangle_{\beta}\langle W(0) W(0)\rangle_{\beta}}=1-\kappa g^{2} e^{\lambda_{L} t} \quad \text { for } \quad t_{d}<t<t_{*} .
$$

Here, $g^{2}$ is proportional to the inverse of the large central charge $c$, and $\kappa$ is a constant depending on the details of models. Note that the scrambling time $t_{*}$ is of order $\frac{1}{\lambda_{L}} \log \frac{1}{g^{2}}$. The exponential growth rate $\lambda_{L}$ is known as the Lyapunov exponent. It was shown [5] that the Lyapunov exponent $\lambda_{L}$ is bounded in a quantum field theory with unitarity and causality. ${ }^{1}$ I.e.

$$
\lambda_{L} \leqq \frac{2 \pi}{\beta} .
$$

This bound on chaos indicates the concept of the maximal chaos, and it was shown that SYK models [7-19], the tensor models [16, 20-24], the dilaton gravity on nearly- $\mathrm{AdS}_{2}$ [25]

\footnotetext{
${ }^{1}$ Also, see [6] for understanding of the bound on chaos from the stability of the Schwarzian theory.
} 
and string worldsheet theories [26-28] have the maximal Lyapunov exponent $\lambda_{L}=\frac{2 \pi}{\beta}$. In such a maximally chaotic system, (1.2) is almost constant in early time because the exponential growth is negligible compared to the leading constant term. As time is increased, the sub-leading exponential growth with $\lambda_{L}=\frac{2 \pi}{\beta}$ in (1.2) is comparable to the leading constant around the scrambling time $t_{*} \sim \frac{1}{\lambda_{L}} \log \frac{1}{g^{2}}$. At the same time, the next sub-leading term of order $\mathcal{O}\left(g^{4}\right)$ would also become of importance. Then, it is interesting to ask a question whether the next sub-leading correction of order $\mathcal{O}\left(g^{4}\right)$ increases or decreases the maximum Lyapunov exponent $\lambda_{L}=\frac{2 \pi}{\beta}$.

In this paper, we will make an attempt to answer this question ${ }^{2}$ in the Schwarzian theory [35-41] which describes the low-energy sector of the SYK-like models and the dilaton gravity on the nearly- $\mathrm{AdS}_{2}$. And, it is responsible for the saturation of the bound on chaos thereof. Although not all the quantum correction of order $\mathcal{O}\left(g^{4}\right) \sim \mathcal{O}\left(\frac{1}{c^{2}}\right)$ to the OTOC exhibits universality, the contribution of the Schwarzian modes of order $\mathcal{O}\left(g^{4}\right)$ will be universal.

The outline of this paper is as follows. In section 2, we review the semi-classical analysis of the Schwarzian theory. Then, we evaluate the propagator of the Schwarzian soft mode and its loop correction. In section 3, we consider a bi-local field and its Euclidean two point function which corresponds to four point function of a fundamental local field. By studying the soft mode expansion of the dressed bi-locals, we evaluate the contribution of the soft mode to the Euclidean two point function of bi-locals. Then, taking analytic continuation to the real time, we obtain the soft mode contribution to OTOC. In section 4, we make concluding remarks, and we present caveats and future directions.

\section{Schwarzian theory}

\subsection{Review}

We begin with the review of the Schwarzian theory in [35]. The partition function of the Schwarzian theory is given by

$$
Z[g]=\int \frac{\mu[\phi]}{\mathrm{SL}(2, \mathbb{R})} \exp \left[-\frac{1}{2 g^{2}} \int_{0}^{2 \pi} d \tau\left(\left(\frac{\phi^{\prime \prime}}{\phi^{\prime}}\right)^{2}-\left(\phi^{\prime}\right)^{2}\right)\right],
$$

where $\phi(\tau) \in \operatorname{Diff}\left(S^{1}\right)$ is a diffeomorphism of a circle. The Schwarzian theory has $\operatorname{SL}(2, \mathbb{R})$ symmetry given by

$$
\tan \frac{\phi}{2} \longrightarrow \frac{a \tan \frac{\phi}{2}+b}{c \tan \frac{\phi}{2}+d} \quad(a, b, c, d \in \mathbb{R} \quad \text { and } \quad a d-b c=1)
$$

and we mod out the $\mathrm{SL}(2, \mathbb{R})$ volume. Hence, the physical degree of freedom of the Schwarzian theory lives on the quotient space $\operatorname{Diff}\left(S^{1}\right) / \operatorname{SL}(2)$. Note that $\mu[\phi]$ is the reparametrization invariant measure. After $\mathrm{SL}(2, \mathbb{R})$ gauge-fixing, the measure

\footnotetext{
${ }^{2}$ Note that there has been a series of works to pursue a similar question [29-31]. Also, see [32-34].
} 
becomes $[35,42,43]$

$$
\mu[\phi] \equiv \frac{\mathcal{D} \phi}{\prod_{\tau} \phi^{\prime}(\tau)} .
$$

The measure $\mu[\phi]$ can be exponentiated by introducing a fermion $\psi(\tau)$, and the partition function of the Schwarzian in (2.1) can be written as [35]

$$
Z[g]=\int \frac{\mathcal{D} \phi \mathcal{D} \psi}{\operatorname{SL}(2, \mathbb{R})} e^{-S}
$$

where the action $S$ is given by

$$
S=\frac{1}{2} \int_{0}^{2 \pi} d \tau\left[\frac{1}{g^{2}}\left(\frac{\phi^{\prime \prime}}{\phi^{\prime}}\right)^{2}-\frac{1}{g^{2}}\left(\phi^{\prime}\right)^{2}+\frac{\psi^{\prime \prime} \psi^{\prime}}{\left(\phi^{\prime}\right)^{2}}-\psi^{\prime} \psi\right]
$$

In the weak coupling limit $g \ll 1$, one can perform the semi-classical analysis of the action in (2.5) by expanding the diffeomorphism $\phi(\tau)$ around a saddle point $\phi(\tau)=\tau$ :

$$
\phi(\tau)=\tau+g \epsilon(\tau)
$$

Accordingly, the Schwarzian action in (2.5) can be expanded with respect to $g$ :

$$
S=-\frac{\pi}{g^{2}}+S^{(2)}+g S^{(3)}+g^{2} S^{(4)}+\mathcal{O}\left(g^{3}\right),
$$

where we have

$$
\begin{aligned}
& S^{(2)}=\frac{1}{2} \int_{0}^{2 \pi} d \tau\left[\left(\epsilon^{\prime \prime}\right)^{2}-\left(\epsilon^{\prime}\right)^{2}+\psi^{\prime \prime} \psi^{\prime}-\psi^{\prime} \psi\right], \\
& S^{(3)}=\frac{1}{2} \int_{0}^{2 \pi} d \tau \epsilon^{\prime}\left[-2\left(\epsilon^{\prime \prime}\right)^{2}-2 \psi^{\prime \prime} \psi^{\prime}\right], \\
& S^{(4)}=\frac{1}{2} \int_{0}^{2 \pi} d \tau\left(\epsilon^{\prime}\right)^{2}\left[3\left(\epsilon^{\prime \prime}\right)^{2}+3 \psi^{\prime \prime} \psi^{\prime}\right] .
\end{aligned}
$$

After fixing the SL(2) gauge [35]

$$
\int d \tau \epsilon(\tau)=\int d \tau e^{ \pm i \tau} \epsilon(\tau)=0 \quad, \quad \int d \tau \psi(\tau)=\int d \tau e^{ \pm i \tau} \psi(\tau)=0
$$

we Fourier-transform the soft mode $\epsilon(\tau)$ and the fermion $\psi(\tau)$ to the (discrete) momentum space:

$$
\epsilon(\tau)=\sum_{|n| \geqq 2} \epsilon_{n} e^{-i n \tau} \quad, \quad \psi(\tau)=\sum_{|n| \geqq 2} \psi_{n} e^{-i n \tau}
$$




$$
\left\langle\epsilon_{-n} \epsilon_{n}\right\rangle_{\text {free }}=\text { mm }\left\langle\psi_{-n} \psi_{n}\right\rangle_{\text {free }}=
$$
(a) Soft mode
(b) Fermion

Figure 1. Free propagator of the Schwarzian soft mode and the fermion.

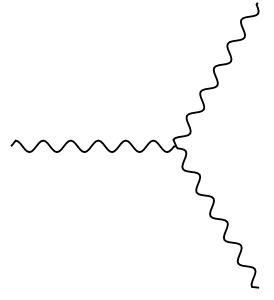

(a) Cubic vertex

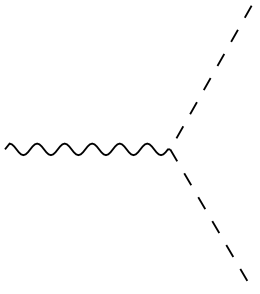

(b) Fermi cubic vertex

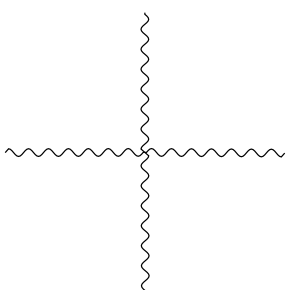

(c) Quartic vertex

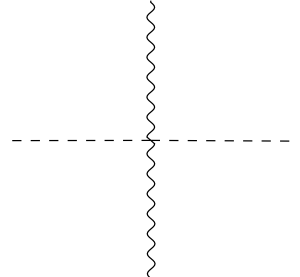

(d) Fermi quartic vertex

Figure 2. Vertices.

In the momentum space, $(2.8) \sim(2.10)$ can be written as

$$
\begin{aligned}
S^{(2)}= & \pi \sum_{|n| \geqq 2} n^{2}\left(n^{2}-1\right) \epsilon_{-n} \epsilon_{n}-\pi i \sum_{|n| \geqq 2} n\left(n^{2}-1\right) \psi_{-n} \psi_{n} \\
S^{(3)}= & \frac{2 \pi i}{3} \sum_{\substack{|n|,|m| \geqq 2 \\
|m+n| \geqq 2}} m n(m+n)\left(m^{2}+m n+n^{2}\right) \epsilon_{-m-n} \epsilon_{m} \epsilon_{n} \\
& +\pi \sum_{\substack{|n|,|m| \geqq 2 \\
|m+n| \geqq 2}} n m(m+n)(n+2 m) \psi_{-m-n} \psi_{m} \epsilon_{n} \\
S^{(4)}= & -\frac{\pi}{2} \sum_{\substack{|n|,|m|,|p| \geqq 2 \\
|m+n+p| \geqq 2}} m n p(m+n+p)\left(m^{2}+n^{2}+p^{2}+m n+n p+p m\right) \epsilon_{-m-n-p} \epsilon_{m} \epsilon_{n} \epsilon_{p} \\
& +\frac{3 \pi i \sum_{\substack{|n|,|m|,|p| \geqq 2 \\
|m+n+p| \geqq 2}} m n p(m+n+p)(m+n+2 p) \psi_{-m-n-p} \psi_{p} \epsilon_{m} \epsilon_{n}}{}
\end{aligned}
$$

They give the free propagator as well as cubic, quartic vertices of the soft modes and fermions (see figure $1 \sim 2$ ). For the leading quantum correction to OTOC, it is enough to consider the interactions up to quartic vertex.

\subsection{Soft mode propagator}

From the quadratic action in (2.13), one can read off the free propagator of the soft mode and the fermion in (discrete) momentum space:

$$
\left\langle\epsilon_{-n} \epsilon_{n}\right\rangle_{\text {free }}=\frac{1}{2 \pi} \frac{1}{n^{2}\left(n^{2}-1\right)} \quad, \quad\left\langle\psi_{-n} \psi_{n}\right\rangle_{\text {free }}=\frac{1}{2 \pi i} \frac{1}{n\left(n^{2}-1\right)} .
$$




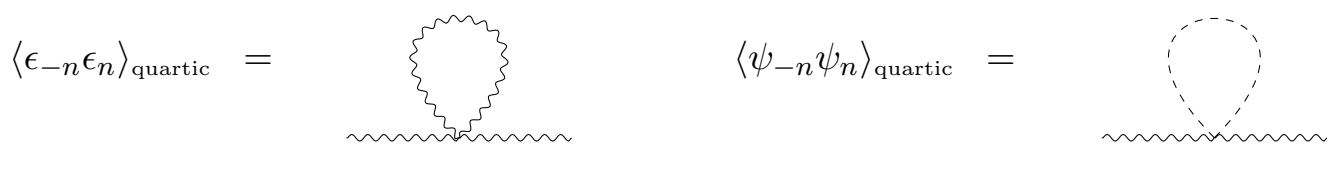

$\begin{array}{ll}\text { (a) Soft mode loop } & \text { (b) Fermi loop }\end{array}$

Figure 3. The loop correction by one quartic vertex to the propagator.

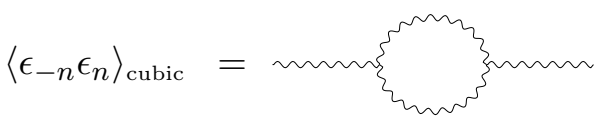

(a) Soft mode loop

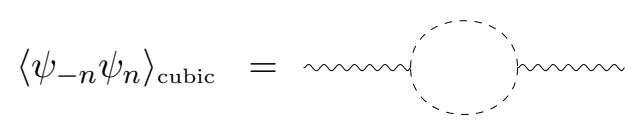

(b) Fermi loop

Figure 4. The loop correction by two cubic vertices to the propagator.

Now, we will evaluate the loop correction of order $\mathcal{O}\left(g^{2}\right)$ to the propagators in (2.16). At order $\mathcal{O}\left(g^{2}\right)$, there are two types of loops: one with a quartic vertex and one with two cubic vertices. And, each loop correction gives divergence. However, as in the calculation of the free energy [35], the divergence in the bosonic loop is cancelled with that of the fermion loop of the same type, which leads to a finite answer.

One-loop with one quartic vertex: we evaluate the contribution of the two diagrams in figure 3 with bosonic and fermi loops made of quartic vertex:

$$
\left\langle\epsilon_{-n} \epsilon_{n}\right\rangle_{\text {quartic }}=-\frac{6 g^{2}}{8 \pi^{2}} \sum_{|m| \geqq 2} \frac{n^{2}+m^{2}}{n^{2}\left(n^{2}-1\right)^{2}\left(m^{2}-1\right)}+\frac{6 g^{2}}{8 \pi^{2}} \sum_{|m| \geqq 2} \frac{m^{2}}{n^{2}\left(n^{2}-1\right)^{2}\left(m^{2}-1\right)} .
$$

Note that each series does not converge because the term of each series does not converge to zero as $m$ goes to infinity. However, the summation of two terms converges to zero as $m \rightarrow \infty$, and its series can be expressed as contour integral as follow.

$$
\begin{aligned}
\left\langle\epsilon_{-n} \epsilon_{n}\right\rangle_{\text {quartic }} & =-\frac{3 g^{2}}{4 \pi^{2}} \sum_{|m| \geqq 2} \frac{1}{\left(m^{2}-1\right)\left(n^{2}-1\right)^{2}}, \\
& =-\frac{3 g^{2}}{4 \pi^{2}} \frac{1}{\left(n^{2}-1\right)^{2}} \frac{1}{2 \pi i} \oint_{\mathcal{C}} d \zeta \frac{\pi}{\tan \pi \zeta} \frac{1}{\left(\zeta^{2}-1\right)} .
\end{aligned}
$$

where the contour $\mathcal{C}$ is a collection of small counterclockwise circles centered at $\zeta \in$ $\mathbb{Z} /\{-1,0,1\}$. By deforming the contour, it can be changed into the sum of the residues at $\zeta=-1,0,1$ :

$$
\left\langle\epsilon_{-n} \epsilon_{n}\right\rangle_{\text {quartic }}=\frac{3 g^{2}}{4 \pi^{2}} \frac{1}{\left(n^{2}-1\right)^{2}} \sum_{m=-1,0,1} \operatorname{Res}_{\zeta=m} \frac{\pi}{\tan \pi \zeta} \frac{1}{\left(\zeta^{2}-1\right)}=-\frac{9 g^{2}}{8 \pi^{2}} \frac{1}{\left(n^{2}-1\right)^{2}} .
$$


One-loop with two cubic vertices: in a similar way, one can calculate the sum of two diagrams with bosonic and fermi loop composed of two cubic vertices in figure 4. Again, each diagram does not converge, but the summation of the two diagram gives a finite contribution.

$$
\begin{aligned}
\left\langle\epsilon_{-n} \epsilon_{n}\right\rangle_{\text {cubic }}= & \frac{g^{2}}{2 \pi^{2}} \sum_{\substack{|m| \geqq 2 \\
|m+n| \geqq 2}} \frac{\left(m^{2}+m n+n^{2}\right)^{2}}{n^{2}\left(n^{2}-1\right)^{2}\left(m^{2}-1\right)\left((m+n)^{2}-1\right)} \\
& -\frac{g^{2}}{8 \pi^{2}} \sum_{\substack{|m| \geqq 2 \\
|m+n| \geqq 2}} \frac{m(n+m)(n+2 m)^{2}}{n^{2}\left(n^{2}-1\right)^{2}\left(m^{2}-1\right)\left((m+n)^{2}-1\right)}, \\
= & \frac{g^{2}}{8 \pi^{2}} \sum_{\substack{|m| \geqq 2 \\
|m+n| \geqq 2}} \frac{7 m^{2}+7 m n+4 n^{2}}{\left(n^{2}-1\right)^{2}\left(m^{2}-1\right)\left((m+n)^{2}-1\right)} .
\end{aligned}
$$

It can also be written as contour integral as before.

$$
\left\langle\epsilon_{-n} \epsilon_{n}\right\rangle_{\text {cubic }}=\frac{g^{2}}{8 \pi^{2}} \sum_{\substack{|m| \geqq 2 \\|m+n| \geqq 2}} \frac{1}{\left(n^{2}-1\right)^{2}} \frac{1}{2 \pi i} \oint_{\mathcal{C}} \frac{\pi}{\tan \pi \zeta} \frac{7 \zeta^{2}+7 \zeta n+4 n^{2}}{\left(\zeta^{2}-1\right)\left((\zeta+n)^{2}-1\right)} .
$$

However, one has to consider the contour for $|n|>2$ case and for $n=2$ case separately. For the case of $|n|>2$, the contour $\mathcal{C}$ is a collection of small counterclockwise circles centered at $\zeta \in \mathbb{Z} /\{-n-1,-n,-n+1,-1,0,1\}$. For $n=2$, the contour $\mathcal{C}$ is a collection of small counterclockwise circles around integers except for $\{-3,-2,-1,0,1\}$ i.e., $\zeta \in$ $\mathbb{Z} /\{-3,-2,-1,0,1\}$, and similar for $n=-2$. (See figure 7 ) By deforming the contour, we pick up the residue at the rest of the poles, and we have

$$
\left\langle\epsilon_{-n} \epsilon_{n}\right\rangle_{\text {cubic }}=\left\{\begin{array}{ll}
\frac{g^{2}}{8 \pi^{2}} \frac{-112+104 n^{2}+179 n^{4}-111 n^{6}+12 n^{8}}{n^{2}\left(n^{2}-4\right)^{2}\left(n^{2}-1\right)^{3}} & \text { for } n \neq \pm 2 \\
-\frac{g^{2}}{96}+\frac{1465 g^{2}}{6912 \pi^{2}} & \text { for } n= \pm 2
\end{array} .\right.
$$

\section{Out-of-time-ordered correlator}

We will evaluate the contribution of the Schwarzian soft mode to OTOC. For this, we first evaluate Euclidean four point function in a specific configuration, and then we will take analytic continuation to real time OTOC.

\subsection{Dressed bi-local field}

The OTOC is basically a four point function of "matter" fields. In the SYK model, the OTOC of the fundamental fermion $\chi^{i}(\tau)(i=1,2, \cdots, N)$ was evaluated $[12,16-$ $18,44]$. This can also be viewed as two point function of the bi-local field $\psi\left(\tau_{1}, \tau_{2}\right) \equiv$ $\frac{1}{N} \sum_{i=1}^{N} \chi^{i}\left(\tau_{1}\right) \chi^{i}\left(\tau_{2}\right)$. A similar bi-local field from a matter scalar field was used to evaluated OTOC in the two-dimensional dilaton gravity on the nearly-AdS 2 [25]. Here, the 
gravity sector is described by Schwarzian action, and the scalar matter field was included on top of the Schwarzian mode. On the other hand, without coupling to an extra matter field, one can also construct the bi-local field by a (boundary-to-boundary) SL(2) Wilson line of BF theory for $\mathrm{AdS}_{2}[39,40,45-47]$ or Chern- Simons gravity for $\mathrm{AdS}_{3}[48,49] .^{3}$ One can consider a smooth fluctuation around the constant background (e.g., BTZ black hole) with a fixed holonomy along the time circle. This fluctuation can be described by Schwarzian action on the boundary [41, 48, 49, 52]. For OTOC, one can study Wilson line evaluated with the SL(2) gauge field corresponding to the smoothly fluctuated background, which can be interpreted as bi-local field dressed by the soft mode [48, 49]. Note that this soft mode generates conformal transformation on the boundary. Hence, the dressed bi-local field can equivalently be obtained by the conformal transformation of the (boundary-toboundary) two point function. Then, one can expand the dressed bi-local field with respect to the soft mode:

$$
\begin{aligned}
\frac{\Phi^{\text {dressed }}\left(\tau_{1}, \tau_{2}\right)}{\Phi_{c l}\left(\tau_{1}, \tau_{2}\right)} \equiv & \frac{\left[\phi^{\prime}\left(\tau_{1}\right) \phi^{\prime}\left(\tau_{2}\right)\right]^{h}}{\left[\sin \frac{1}{2}\left(\phi\left(\tau_{1}\right)-\phi\left(\tau_{2}\right)\right)\right]^{2 h}} / \frac{1}{\left[\sin \frac{\tau_{12}}{2}\right]^{2 h}} \\
= & 1+g \sum_{|n| \geqq 2} \epsilon_{n} e^{-i n \chi} f_{n}^{(1)}(\sigma)+g^{2} \sum_{|m|,|n| \geqq 2} \epsilon_{m} \epsilon_{n} e^{-i(m+n) \chi} f_{m, n}^{(2)}(\sigma) \\
& +g^{3} \sum_{|m|,|n|,|p| \geqq 2} \epsilon_{m} \epsilon_{n} \epsilon_{p} e^{-i(m+n+p) \chi} f_{m, n, p}^{(3)}(\sigma)+\cdots
\end{aligned}
$$

where $h$ is the conformal dimension of the matter field, and $\Phi_{c l}\left(\tau_{1}, \tau_{2}\right)$ is the leading term in the soft mode expansion which corresponds to the two point function in the constant background. Here, we defined the center of time $\chi$ and the relative time $\sigma$ to be

$$
\chi=\frac{1}{2}\left(\tau_{1}+\tau_{2}\right) \quad, \quad \sigma=\frac{1}{2}\left(\tau_{1}-\tau_{2}\right) .
$$

In addition, the soft mode eigenfunction $f_{n}^{(1)}(\sigma)$ and $f_{m, n}^{(2)}(\sigma)$ in $(3.1)$ are found to be

$$
\begin{aligned}
f_{n}^{(1)}(\sigma) \equiv & -2 i h\left[n \cos n \sigma-\frac{\sin n \sigma}{\tan \sigma}\right] \\
f_{m, n}^{(2)}(\sigma) \equiv & -2 h^{2}\left(m \cos m \sigma-\frac{\sin m \sigma}{\tan \sigma}\right)\left(n \cos n \sigma-\frac{\sin n \sigma}{\tan \sigma}\right) \\
& +h\left[m n \cos (m+n) \sigma-\frac{\sin m \sigma \sin n \sigma}{\sin ^{2} \sigma}\right] .
\end{aligned}
$$

In particular, it is convenient to evaluate them at $\sigma=-\frac{\pi}{2}$ :

$$
\begin{aligned}
f_{n}^{(1)}\left(-\frac{\pi}{2}\right) & =-2 i h n \cos \frac{n \pi}{2} \\
f_{m, n}^{(2)}\left(-\frac{\pi}{2}\right) & =-h\left[(2 h-1) m n \cos \frac{m \pi}{2} \cos \frac{n \pi}{2}+(m n+1) \sin \frac{m \pi}{2} \sin \frac{n \pi}{2}\right] .
\end{aligned}
$$

\footnotetext{
${ }^{3}$ For the path integral representation of Wilson line, one need to consider a probe particle moving on the $\operatorname{SL}(2, \mathbb{R})$ group manifold $[50,51]$.
} 
The form of $f_{m, n, p}^{(3)}(\sigma)$ is complicated, but it is enough to evaluate it at a particular value for our purpose.

$$
f_{m, n,-m}^{(3)}\left(-\frac{\pi}{2}\right)=-\frac{1}{3} i h e^{-i n \chi} n \cos \frac{n \pi}{2}\left[h+2(h-1)^{2} m^{2}+h\left(-1+2(h-1) m^{2}\right) \cos m \pi\right] .
$$

\subsection{Euclidean four point function}

In this section, we will evaluate the contribution of the soft mode to the Euclidean two point function of the dressed bi-local fields $\Phi^{\text {dressed }}$, which corresponds to four point function of a matter field:

$$
\left\langle\Phi^{\text {dressed }}\left(\tau_{1}, \tau_{2}\right) \Phi^{\text {dressed }}\left(\tau_{3}, \tau_{4}\right)\right\rangle .
$$

The leading contribution is the product of the one point function of the bi-locals which corresponds to the disconnected diagrams in the four point function of the matters in the constant background.

$$
\left\langle\Phi^{\text {dressed }}\left(\tau_{1}, \tau_{2}\right) \Phi^{\text {dressed }}\left(\tau_{3}, \tau_{4}\right)\right\rangle=\Phi_{c l}\left(\tau_{1}, \tau_{2}\right) \Phi_{c l}\left(\tau_{3}, \tau_{4}\right)+\cdots
$$

where the leading one-point function $\Phi_{c l}\left(\tau_{1}, \tau_{2}\right)$ is given by

$$
\Phi_{c l}\left(\tau_{1}, \tau_{2}\right) \equiv\left\langle\Phi^{\mathrm{dressed}}\left(\tau_{1}, \tau_{2}\right)\right\rangle_{\text {leading }} \sim \frac{1}{\left[\sin \frac{\tau_{12}}{2}\right]^{2 h}} .
$$

Note that one can evaluate the correction to the one point function of the bi-local field. For this, we perform the soft mode expansion of the one point function:

$$
\begin{aligned}
\Phi_{0}^{\text {dressed }}(\sigma) & \equiv \frac{\left\langle\Phi^{\text {dressed }}\left(\tau_{1}, \tau_{2}\right)\right\rangle}{\Phi_{c l}\left(\tau_{1}, \tau_{2}\right)}=1+g^{2} \sum_{|n| \geqq 2}\left\langle\epsilon_{-n} \epsilon_{n}\right\rangle f_{-n, n}^{(2)}(\sigma)+\cdots \\
& =1+\frac{1}{4} g^{2} h\left(4+8 h-\pi^{2}\right)+\cdots
\end{aligned}
$$

Note that $\Phi_{0}^{\text {dressed }}$ is independent of $\chi$ because of SL $(2)$ charge. In this paper, we will consider a fixed value of $\sigma$ (i.e., $\sigma=-\frac{\pi}{2}$ ), and then $\Phi_{0}^{\text {dressed }}$ is nothing but a numerical constant.

One may evaluate the Euclidean four point function for any choice of $\left(\tau_{1}, \tau_{2} ; \tau_{3}, \tau_{4}\right)$. However, for the analytic continuation to a particular OTOC, it is enough to consider the following configuration which simplifies the calculation of the four point function:

$$
\left(\tau_{1}, \tau_{2}, \tau_{3}, \tau_{4}\right)=\left(\chi-\frac{\pi}{2}, \chi+\frac{\pi}{2}, 0, \pi\right)
$$

where $\chi \in(-\pi / 2, \pi / 2)$. Therefore, we will evaluate the following Euclidean four point function.

$$
\mathcal{F}(\chi) \equiv \frac{\left\langle\Phi^{\mathrm{dressed}}(\chi-\pi / 2, \chi+\pi / 2) \Phi^{\mathrm{dressed}}(0, \pi)\right\rangle}{\Phi_{c l}(\chi-\pi / 2, \chi+\pi / 2) \Phi_{c l}(0, \pi)}=\mathcal{F}_{d}+g^{2} \mathcal{F}^{(1)}(\chi)+g^{4} \mathcal{F}^{(2)}(\chi)+\mathcal{O}\left(g^{6}\right),
$$

where the numerical value $\mathcal{F}_{d}=\left[\Phi_{0}^{\text {dressed }}\left(-\frac{\pi}{2}\right)\right]^{2}$ corresponds to the disconnected diagrams. 
(a) Leading $\sim \mathcal{O}\left(g^{2}\right)$
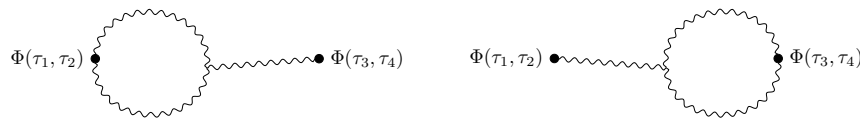

(c) Three soft modes scattering $\sim \mathcal{O}\left(g^{4}\right)$
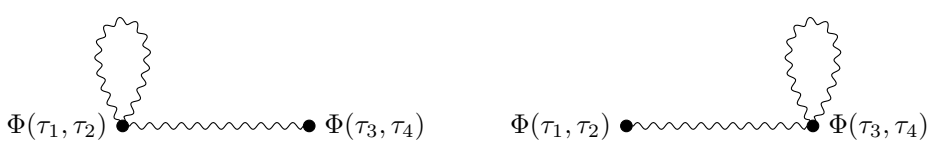

(e) Soft modes dressing $\sim \mathcal{O}\left(g^{4}\right)$

Figure 5. Diagrams for the contributions of the soft mode to the Euclidean two point function of bi-local fields. We represent the dressed bi-local field by a dot.

Leading connected diagram of order $\mathcal{O}\left(\boldsymbol{g}^{2}\right)$ : from the soft mode expansion (3.1), the leading contribution of the soft modes to the (connected) four point function at the configuration (3.12) (See figure $5(\mathrm{a})$ ) is found to be

$$
\mathcal{F}^{(1)}(\chi)=\sum_{|n| \geqq 2}\left\langle\epsilon_{-n} \epsilon_{n}\right\rangle e^{i n \chi} e^{-\frac{n \pi i}{2}} f_{-n}^{(1)}\left(-\frac{\pi}{2}\right) f_{n}^{(1)}\left(-\frac{\pi}{2}\right)=4 h^{2} \sum_{\substack{|n| \geq 2 \\ n: \text { even }}} \frac{e^{i n \chi} e^{-\frac{\pi i n}{2}}}{2 \pi\left(n^{2}-1\right)},
$$

where we used the leading propagator of the soft mode in (2.16). This can be written as the following contour integral

$$
\mathcal{F}^{(1)}(\chi)=4 h^{2} \frac{1}{2 \pi i} \oint_{\mathcal{C}} d \zeta \frac{\frac{\pi}{2}}{\sin \frac{\pi \zeta}{2}} \frac{e^{i \zeta \chi}}{2 \pi\left(\zeta^{2}-1\right)} .
$$

Here, the contour $\mathcal{C}$ is a collection of small counterclockwise circles centered at $\zeta \in$ $\mathbb{Z} /\{-1,0,1\}$ (See figure 6). Then, we deform the contour to pick up the rest of poles. I.e.

$$
\mathcal{F}^{(1)}(\chi)=-4 h^{2} \sum_{n=0, \pm 1} \operatorname{Res}_{\zeta=n} \frac{\frac{\pi}{2}}{\sin \frac{\pi \zeta}{2}} \frac{e^{i \zeta \chi}}{2 \pi\left(\zeta^{2}-1\right)}=\frac{2 h^{2}}{\pi}\left(1-\frac{\pi}{2} \cos \chi\right) .
$$

Loop correction of order $\mathcal{O}\left(\boldsymbol{g}^{4}\right)$ : now, let us consider the loop corrections to the soft mode propagator in (2.20) and (2.23) (See figure 5(b)), which give a contribution to $g^{4} \mathcal{F}^{(2)}(\chi)$.

$$
\mathcal{F}_{\text {loop }}^{(2)}(\chi)=4 h^{2} \sum_{\substack{|n| \geq 2 \\ n: \text { even }}} e^{i n \chi} e^{-\frac{\pi i n}{2}}\left\langle\epsilon_{-n} \epsilon_{n}\right\rangle_{\text {loop }} n^{2} \subset \mathcal{F}^{(2)}(\chi)
$$



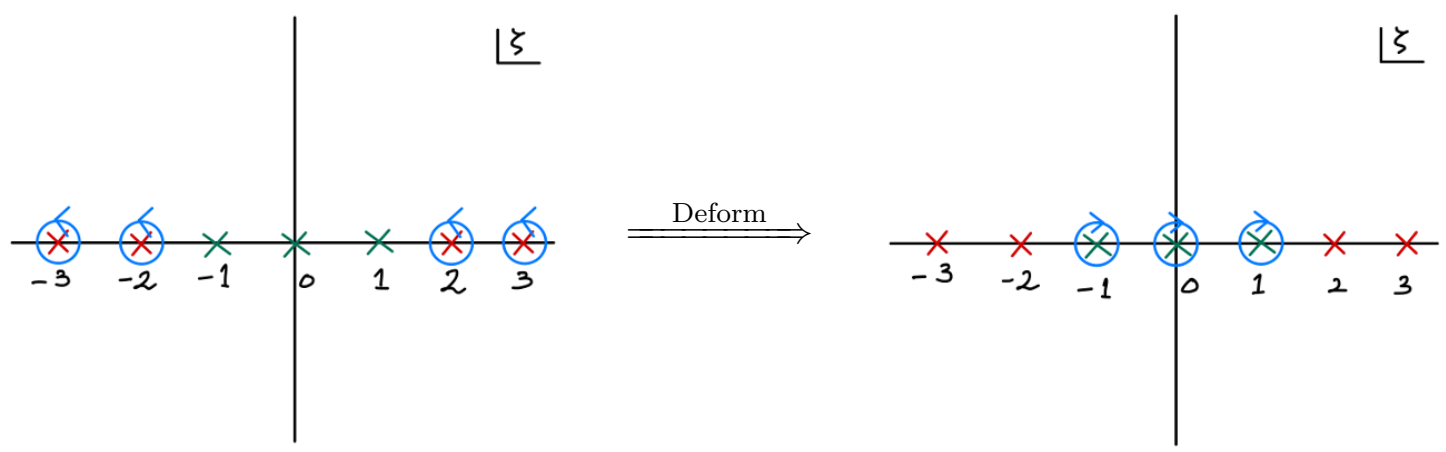

Figure 6. The contribution of the soft mode can be written as the contour integral along circles with small radius centered at $\zeta \in \mathbb{Z} /\{-1,0,1\}$. After deforming the contour, one can rewrite it as the sum of residues at $\zeta=-1,0,1$.

For the loop with one quartic vertex, one can repeat the same calculation as the leading contribution. However, for the loop with two cubic vertices, one has to calculate $|n|>2$ case and $n= \pm 2$ case separately. For $|n|>2$, one can also rewrite the contribution as a contour integral where the contour is a collection of small counterclockwise circles centered at $\zeta \in \mathbb{Z} /\{-2,-1,0,1,2\}$. And, the deformation of the contour gives the sum of residues at $\zeta=-2,-1,0,1,2$. Summing up the results for $|n|>2$ and $n= \pm 2$, we have

$$
\begin{aligned}
\mathcal{F}_{\text {loop }}^{(2)}(\chi)=\frac{7 h^{2}}{2 \pi^{2}}[-1+ & \frac{\pi}{168}\left(88-3 \pi^{2}+12 \chi^{2}\right) \cos \chi+\frac{1}{126}\left(-79+9 \pi^{2}+36 \chi^{2}\right) \cos 2 \chi \\
& \left.+\frac{5 \pi}{14} \chi \sin \chi-\frac{2}{3} \chi \sin 2 \chi\right] .
\end{aligned}
$$

Correction from scattering of three soft modes with cubic vertex of order $\mathcal{O}\left(g^{4}\right)$ : $\mathcal{F}^{(2)}(\chi)$ includes the contribution from the scattering of three soft modes emitted from the bi-locals. Using the cubic vertex in $(2.14)$ and the soft mode eigenfunction $f_{n}^{(1)}(\sigma)$ in $(3.5)$ and $f_{m, n}^{(2)}(\sigma)$ in (3.6), this can be written as follows.

$$
\begin{aligned}
& \mathcal{F}_{\text {three soft modes scattering }}^{(2)} \\
& =-\frac{2 h^{2}}{\pi^{2}} \sum_{\substack{m, n: \text { even } \\
|m|,|n| \geqq 2,|m+n| \geqq 2}} \frac{(2 h-1)\left(m^{2}+m n+n^{2}\right)}{\left(n^{2}-1\right)\left(m^{2}-1\right)\left[(n+m)^{2}-1\right]}(-1)^{\frac{n+m}{2}} \cos (n+m) \chi \\
& \quad-\frac{2 h^{2}}{\pi^{2}} \sum_{\substack{m, n: \text { odd } \\
|m|,|n| \geqq 2,|m+n| \geqq 2}} \frac{(m n+1)\left(m^{2}+m n+n^{2}\right)}{n m\left(n^{2}-1\right)\left(m^{2}-1\right)\left[(n+m)^{2}-1\right]}(-1)^{\frac{n+m}{2}} \cos (n+m) \chi .
\end{aligned}
$$

As in the calculation of the loop with two cubic vertices, one has to evaluate them for $|n|>2$ and $n= \pm 2$ separately because of the constraint $|m+n| \geqq 2$ in the summation 

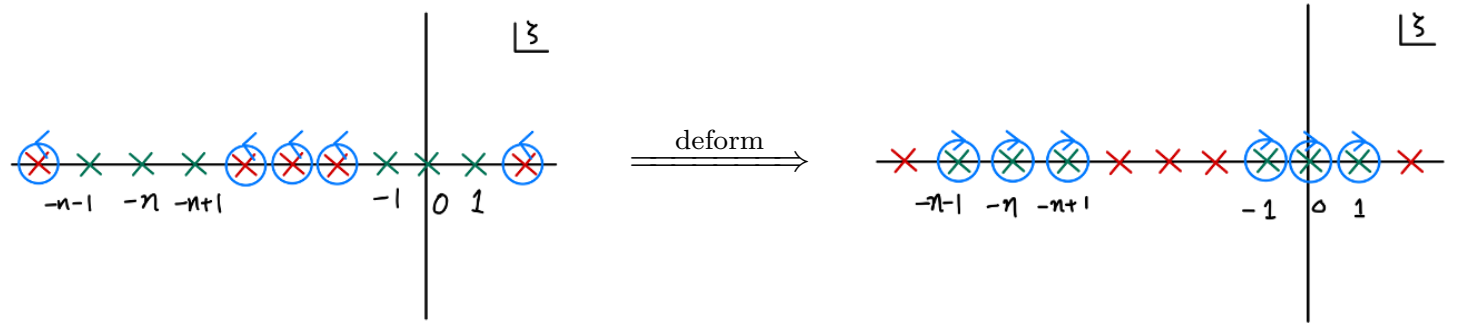

(a) $n \neq \pm 2$
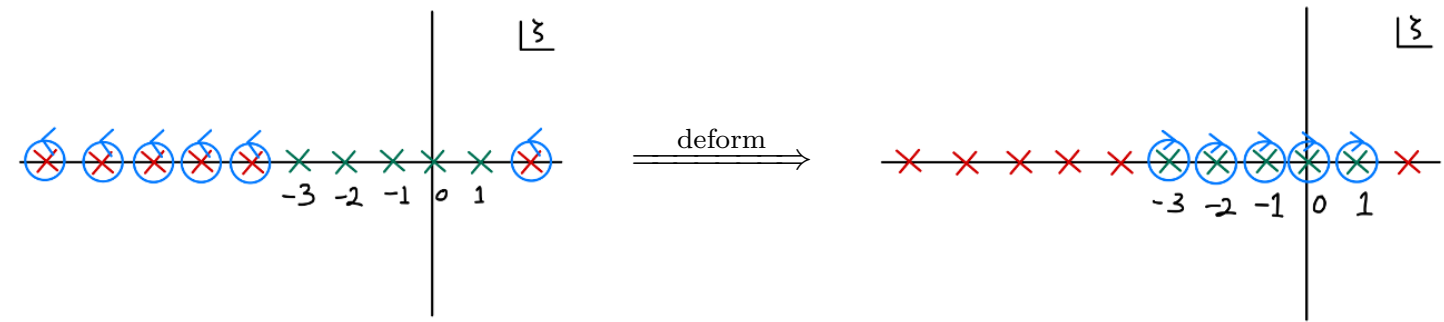

(b) $n=2$

Figure 7. The soft mode contribution can be expressed as the contour integral along circles with small radius centered at $\zeta \in \mathbb{Z} /\{-1,0,1,-n-1,-n,-n+1\}$ for $n \neq \pm 2$, at $\zeta \in \mathbb{Z} /\{-3,-2-1,0,1$, for $n=2$ or at $\zeta \in \mathbb{Z} /\{-1,0,1,2,3\}$ for $n=-2$, respectively. By deforming the contour, it can be expressed as the sum of residues at $\zeta=-n-1,-n,-n+1,-1,0,1, \zeta=-3,-2,-1,0,1$ or $\zeta=-1,0,1,2,3$, respectively.

(See figure 7). We found

$$
\begin{aligned}
\mathcal{F}_{\text {three soft modes scattering }}^{(2)}= & \frac{h^{2}}{\pi^{2}}\left[-1+\frac{1}{36}\left(178+9(2 h-1) \pi^{2}-72 \chi^{2}\right) \cos 2 \chi\right. \\
& \left.+\frac{\pi}{8}\left(-16 h+\pi^{2}-4 \chi^{2}\right) \cos \chi+\pi(2 h-1) \chi \sin \chi+\frac{16}{3} \chi \sin 2 \chi\right] .
\end{aligned}
$$

Correction from two soft modes exchanges of order $\mathcal{O}\left(g^{4}\right)$ : the exchange of two soft modes gives the contribution of order $\mathcal{O}\left(g^{4}\right)$ (See figure $5(\mathrm{~d})$ ). For this, using the soft mode eigenfunction $f_{m, n}^{(2)}(\sigma)$ in (3.6), one can evaluate the diagram for the two soft mode exchange in a similar way.

$$
\begin{aligned}
\mathcal{F}_{\text {two soft modes exchange }}^{(2)}= & \frac{h^{2}(2 h-1)^{2}}{2 \pi^{2}} \sum_{\substack{n, m: \text { even } \\
|m|,|n| \geqq 2}} \frac{e^{-i(m+n) \chi} e^{\frac{\pi i}{2}(m+n)}}{\left(n^{2}-1\right)\left(m^{2}-1\right)} \\
& +\frac{h^{2}}{2 \pi^{2}} \sum_{\substack{n, m: \text { odd } \\
|m|,|n| \geqq 2}} \frac{(m n+1)^{2} e^{-i(m+n) \chi} e^{\frac{\pi i}{2}(m+n)}}{n^{2} m^{2}\left(n^{2}-1\right)\left(m^{2}-1\right)} \\
= & \frac{h^{2}}{8 \pi^{2}}\left[4+\pi^{2}\left(-2+\chi^{2}\right)+4 \pi\left(3+\chi^{2}\right) \cos \chi+(2 h-1)^{2}(-2+\pi \cos \chi)^{2}\right. \\
& \left.\quad+\left(-22+8 \chi^{2}\right) \cos 2 \chi-2 \pi \chi \sin \chi-24 \chi \sin 2 \chi\right] .
\end{aligned}
$$



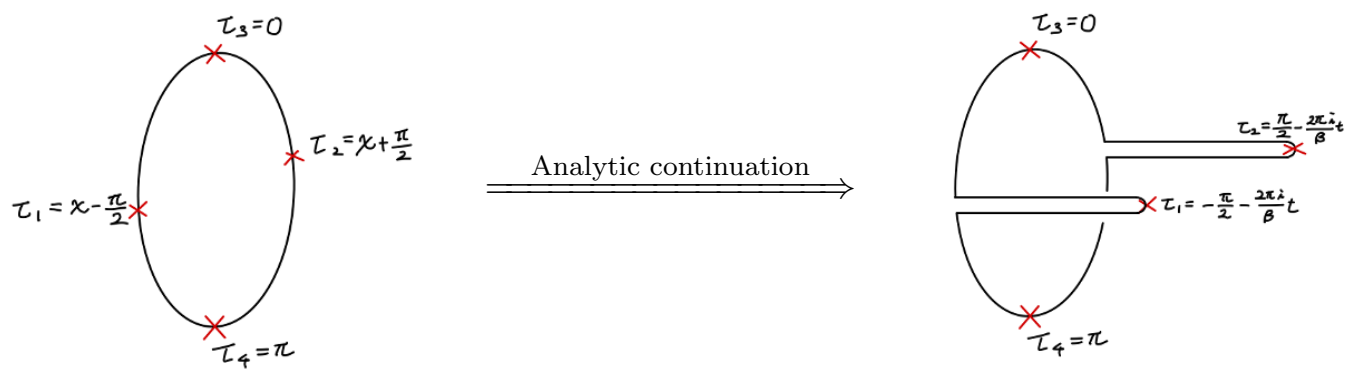

Figure 8. Analytic continuation from the configuration on Euclidean thermal circle to the real time out-of-time-ordered configuration.

Correction from soft mode dressing of order $\mathcal{O}\left(g^{4}\right)$ : one can also consider a correction to the leading diagram in figure 5 (a) by the soft mode dressing. (See figure 5(e)). This contribution of order $\mathcal{O}\left(g^{4}\right)$ can be written as

$$
\mathcal{F}_{\text {soft mode dressing }}^{(2)}=2 \sum_{|n|,|m| \geqq 2} e^{i n \chi} e^{-\frac{n \pi i}{2}} f_{m, n,-m}^{(3)}\left(-\frac{\pi}{2}\right) f_{-n}^{(1)}\left(-\frac{\pi}{2}\right)\left\langle\epsilon_{-n} \epsilon_{n}\right\rangle\left\langle\epsilon_{-m} \epsilon_{m}\right\rangle .
$$

In the same way as before, the contour integral representation for the summation over $m$ gives the leading connected diagram in (3.14) (but, of order $\mathcal{O}\left(g^{4}\right)$ ) up to numerical constant. i.e.,

$$
\mathcal{F}_{\text {soft mode dressing }}^{(2)}=\frac{h}{36 \pi}\left(3+6 h-\pi^{2}\right) \sum_{|n| \geqq 2} e^{i n \chi} f_{-n}^{(1)}\left(-\frac{\pi}{2}\right) f_{n}^{(1)}\left(-\frac{\pi}{2}\right)\left\langle\epsilon_{-n} \epsilon_{n}\right\rangle .
$$

Hence, we have

$$
\mathcal{F}_{\text {soft mode dressing }}^{(2)}=\frac{h^{3}}{18 \pi^{2}}\left(3+6 h-\pi^{2}\right)\left(1-\frac{\pi}{2} \cos \chi\right) .
$$

Total contribution of soft modes: summing up the above all results, we have

$$
\begin{aligned}
\mathcal{F}_{\text {total }}(\chi)= & \mathcal{F}_{d}+g^{2} \mathcal{F}^{(1)} \\
& +g^{4}\left[\mathcal{F}_{\text {loop }}^{(2)}+\mathcal{F}_{\text {three soft modes scattering }}^{(2)}+\mathcal{F}_{\text {two soft modes exchange }}^{(2)}+\mathcal{F}_{\text {soft mode dressing }}^{(2)}\right] \\
= & \mathcal{F}_{d}+\frac{2 g^{2} h^{2}}{\pi}\left(1-\frac{\pi}{2} \cos \chi\right) \\
+ & \frac{g^{4} h^{2}}{144 \pi^{2}}\left[288 \pi h \chi \sin \chi+9 \pi^{2}(1+2 h)^{2} \cos 2 \chi\right. \\
& +\pi\left[36 \chi^{2}-4 h\left(3+78 h-\pi^{2}\right)+9\left(24+\pi^{2}\right)\right] \cos \chi \\
& \left.+18 \pi^{2} \chi^{2}+12 h^{2}\left(28+3 \pi^{2}\right)-44 h\left(6+\pi^{2}\right)-504-27 \pi^{2}\right] .
\end{aligned}
$$

\subsection{Real-time out-of-time-ordered correlator}

From the Euclidean four point function in (3.25), we take the analytic continuation from Euclidean time $\chi$ to Lorentzian time $t$ (See figure 8 )

$$
\chi \quad \longrightarrow-\frac{2 \pi i t}{\beta},
$$


in order to obtain the OTOC:

$$
\begin{aligned}
\mathcal{F}_{\text {Отос }}(t)=\mathcal{F}_{d} & -g^{2}\left[\frac{h^{2}}{2} e^{\frac{2 \pi t}{\beta}}-\frac{2 g^{2} h^{2}}{\pi}+\frac{h^{2}}{2} e^{-\frac{2 \pi t}{\beta}}\right] \\
+ & g^{4}\left[\frac{h^{2}(2 h+1)^{2}}{32} e^{\frac{4 \pi t}{\beta}}-\frac{h^{2} \pi}{2 \beta^{2}} t^{2} e^{\frac{2 \pi t}{\beta}}-\frac{2 h^{3}}{\beta} t e^{\frac{2 \pi t}{\beta}}\right. \\
& \left.+\frac{h^{2}\left(312 h^{2}-4 h\left(\pi^{2}-3\right)-9\left(\pi^{2}+24\right)\right)}{288 \pi} e^{\frac{2 \pi t}{\beta}}+\cdots\right],
\end{aligned}
$$

where the ellipsis represents terms that do not grow exponentially at order $\mathcal{O}\left(g^{4}\right)$.

First of all, note that the leading Lyapunov exponent saturates the bound on chaos $[6$, $12,25]$. i.e.,

$$
\lambda_{L}=\frac{2 \pi}{\beta}+\mathcal{O}\left(g^{2}\right) .
$$

Among the contribution of order $\mathcal{O}\left(g^{4}\right)$ in $(3.27)$, a term like $-\frac{2 h^{3}}{\beta} g^{4} t e^{\frac{2 \pi t}{\beta}}$ has been observed to give a correction to the maximum Lyapunov exponent. In SYK model, for instance, the contribution of the non-zero mode to OTOC gives $t e^{\frac{2 \pi t}{\beta}}$ which leads to the $1 / \beta J$ correction to the leading Lyapunov exponent [12]. While the $1 / \beta J$ correction in SYK model decreases the Lyapunov exponent from the maximum value $\frac{2 \pi}{\beta}$, the $1 / c$ correction to the Lyapunov exponent from the Virasoro conformal block in large $c$ was shown [29, 30] to increase the Lyapunov exponent. i.e., $\lambda_{L}=\frac{2 \pi}{\beta}\left(1+\frac{12}{c}\right)$. In our result, the contribution $-\frac{2 h^{3}}{\beta} g^{4} t e^{\frac{2 \pi t}{\beta}}$ in (3.27) seemingly plays a role of increasing the Lyapunov exponent. However, we have other terms at order $\mathcal{O}\left(g^{4}\right)$ which grow faster than $t e^{\frac{2 \pi t}{\beta}}$. In particular, the fastest growing term, $\frac{h^{2}(2 h+1)^{2}}{32} e^{\frac{4 \pi t}{\beta}}$ in (3.27), naively seems to violate the bound on chaos because it grows exponentially with growth rate $\frac{4 \pi}{\beta}$. However, it turns out that it reduces the Lyapunov exponent because its contribution to the OTOC has opposite sign to the leading exponential growth.

Note that each contribution of order $\mathcal{O}\left(g^{4}\right)$ (e.g., the analytic continuation of (3.18), (3.20), (3.21)) includes exponentially growing terms such as $t^{2} e^{\frac{4 \pi t}{\beta}}$ and $t e^{\frac{4 \pi t}{\beta}}$ which grow faster than $e^{\frac{4 \pi t}{\beta}}$. In particular, those in (3.18) and (3.21) play a role of increasing the Lyapunov exponent. On the other hand, the analogous terms in (3.20) decreases the Lyapunov exponent. It is interesting that those fast growing contributions are cancelled exactly, and in the end the remaining fastest growth $e^{\frac{4 \pi t}{\beta}}$ decreases the Lyapunov exponent as we have seen. If we considered only the loop correction in (3.18) for the calculation of OTOC, we would get

$$
\begin{aligned}
& \mathcal{F}_{\text {OтОс } \text { lоö }}(t)=\mathcal{F}_{d}- g^{2}\left[\frac{h^{2}}{2} e^{\frac{2 \pi t}{\beta}}-\frac{2 g^{2} h^{2}}{\pi}+\frac{h^{2}}{2} e^{-\frac{2 \pi t}{\beta}}\right] \\
&+g^{4}\left[-\frac{2 h^{2}}{\beta^{2}} t^{2} e^{\frac{4 \pi t}{\beta}}+\frac{7 h^{2}}{3 \pi \beta} t e^{\frac{4 \pi t}{\beta}}+\frac{h^{2}\left(-79+9 \pi^{2}\right)}{72 \pi^{2}} e^{\frac{4 \pi t}{\beta}}-\frac{h^{2} \pi}{2 \beta^{2}} t^{2} e^{\frac{2 \pi t}{\beta}}\right. \\
&\left.\quad-\frac{5 h^{2}}{4 \beta} t e^{\frac{2 \pi t}{\beta}}-\frac{h^{2}\left(-88+3 \pi^{2}\right)}{96 \pi} e^{\frac{2 \pi t}{\beta}}+\cdots\right]
\end{aligned}
$$

One can easily see that the fastest exponential growth at of order $\mathcal{O}\left(g^{4}\right)$ increases the Lyapunov exponent. 


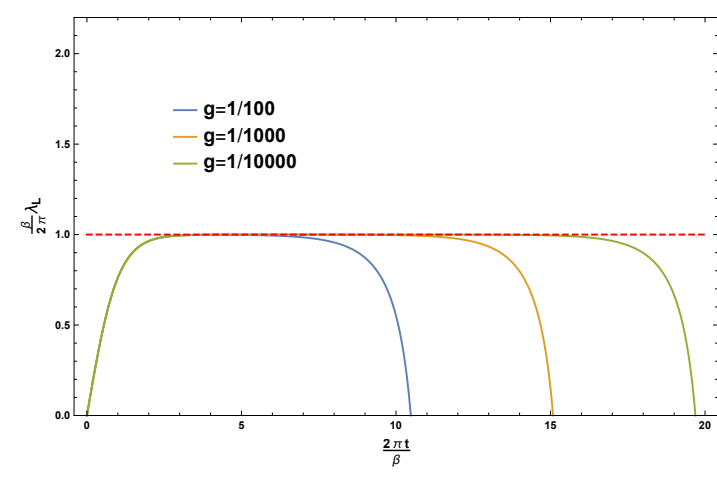

(a) Total contribution

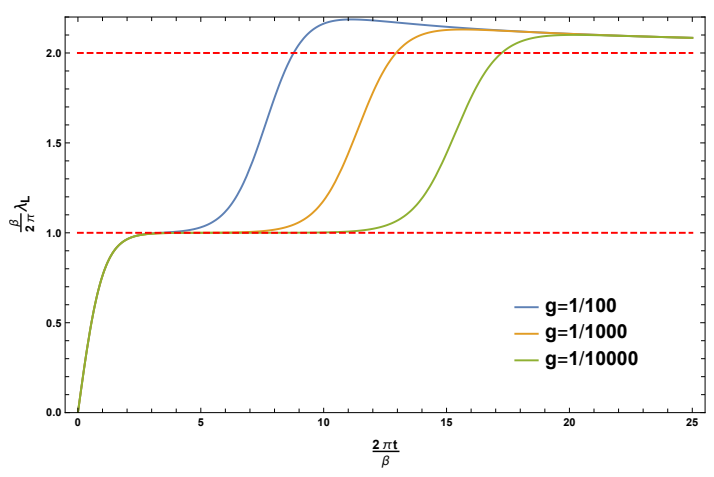

(b) Loop contribution

Figure 9. Lyapunov exponent $\lambda_{L}(t)$ from the total contribution to OTOC and from the loop contribution to OTOC. We plot them for the case of $g=1 / 100,1 / 1000,1 / 10000$, and the corresponding scrambling time would be $\frac{2 \pi}{\beta} t \sim \log g^{2} \simeq 9.21,13.82,18.42$.

To see the change of the Lyapunov exponent concretely, we go back to the original statement of the bound on chaos where we define the Lyapunov exponent by

$$
\lambda_{L}(t) \equiv \frac{d}{d t}\left[\log \left(\mathcal{F}_{\text {const }}-\mathcal{F}(t)\right)\right]
$$

where $\mathcal{F}_{\text {const }}$ corresponds to the constant terms ${ }^{4}$ in $\mathcal{F}(t)$. The bound on chaos states

$$
\lambda_{L}(t) \leqq \frac{2 \pi}{\beta} \quad \text { for } \quad \beta<t<t_{*},
$$

where $t_{*} \sim \frac{\beta}{2 \pi} \log \frac{1}{g^{2}}$ is the scrambling time.

We plot numerically the Lyapunov exponent $\lambda_{L}(t)$ as a function of time from $\mathcal{F}_{\text {Отос }}(t)$ in (3.27) and from $\mathcal{F}_{\text {отос, loop }}(t)$ in (3.29), respectively. See figure 9. Here, we plot the Lyapunov exponent $\lambda_{L}(t)$ for $g=1 / 100,1 / 1000,1 / 10000$ of which the scrambling time would be $\frac{2 \pi}{\beta} t \sim \log g^{2} \simeq 9.21,13.82,18.42$, respectively. In figure 9 (a), the Lyapunov exponent $\lambda_{L}(t)$ from $\mathcal{F}_{\text {отос }}(t)$ in $(3.27)$ is less than $\frac{2 \pi}{\beta}$. As time increase, the Lyapunov exponent quickly saturates the bound, and it begins to decrease around the scrambling time. The term $\frac{g^{4} h^{2}(2 h+1)^{2}}{32} e^{\frac{4 \pi t}{\beta}}$ at order $\mathcal{O}\left(g^{4}\right)$ in (3.27) is responsible for this decrease of Lyapunov exponent. The Lyapunov exponent seemingly vanishes beyond the scrambling time $t_{*}$. However, we cannot trust the Lyapunov exponent beyond the scrambling time because the small $g$ perturbation will break down.

On the other hand, if we had only the loop correction for the quantum correction to the OTOC, we would observe the violation of chaos bound before the scrambling time $t_{*}$. See figure 9(b). This violation of the bound mainly comes from the fastest exponential growth $-g^{4} \frac{2 h^{2}}{\beta^{2}} t^{2} e^{\frac{4 \pi t}{\beta}}$ at order $\mathcal{O}\left(g^{4}\right)$ in $(3.29)$.

\footnotetext{
${ }^{4}$ In the bound on chaos of [5], $\mathcal{F}_{d}$ was used for this constant. However, we choose $\mathcal{F}_{\text {const }}$ to be all constant terms in all order $g$. For example, $\mathcal{F}_{\text {const }}=\mathcal{F}_{d}+\frac{2 g^{2} h^{2}}{\pi}+\mathcal{O}\left(g^{4}\right)$.
} 


\section{Conclusion}

In this paper, we have evaluated the quantum correction of order $\mathcal{O}\left(g^{4}\right)$ by the Schwarzian soft mode to the OTOC. As is well known, the OTOC at order $\mathcal{O}\left(g^{2}\right)$ grows exponentially with the maximum growth rate $\frac{2 \pi}{\beta}[6,12,25]$. At order $\mathcal{O}\left(g^{4}\right)$, we have found that the loop correction by the Schwarzian soft modes and the correction by two soft mode exchanges make the OTOC grow faster than the maximal growth. On the other hand, the correction by three soft mode scattering decreases the exponential growth rate. And, we have showed that the total correction slows down the exponential growth of the OTOC.

Note that the contributions of multi soft mode exchanges have been considered in [25, 29]. For two soft mode exchange, [25, 29] obtained a contribution corresponding to the term $\frac{1}{8} g^{4} h^{2}(2 h-1)^{2} \cos ^{2} \chi$ in (3.21). After analytic contribution to real time, this slows down the exponential growth because it has the opposite sign to the contribution of order $\mathcal{O}\left(g^{2}\right)$. However, in (3.21) we have the other contribution $\frac{h^{2}}{\pi^{2}} \chi^{2} \cos 2 \chi$ which is a faster growth in time. Therefore, we concluded that the overall contribution from the two soft mode exchange speeds up the exponential growth.

It is important to issue caveats in our analysis. First of all, we have not shown that the chaos bound would hold beyond the scrambling time, but we have found that the soft mode contribution to the OTOC at order $\mathcal{O}\left(g^{4}\right)$ slows down the exponential growth of order $\mathcal{O}\left(g^{2}\right)$. To see the behavior of the OTOC beyond the scrambling time, one need to go beyond the perturbation, or, at least, the higher order corrections are required to estimate the behavior beyond the scrambling time. Note that our perturbative calculations in $1 / c$ will break down near the scrambling time. But, the resummation of those higher order corrections would allow us to study the behavior of OTOC beyond the scrambling time. We hope to report the higher order calculations in near future. Also, it would be highly interesting to find a constraint on the behavior of OTOC beyond the scrambling time from a simple physical argument.

In addition, our analysis is based on the Schwarzian theory which might not be as universal as other approaches such as the Virasoro conformal block [29-31] or pole-skipping phenomenon [53-59]. Nevertheless, the low energy physics of many interesting models such as SYK-like models and the dilaton gravity on nearly $\mathrm{AdS}_{2}$ is described by Schwarzian action, in which our result can provide the understanding of the quantum correction to the chaos. It is interesting to explore the quantum correction to chaos in the context of "pole-skipping" phenomenon in the $\mathrm{CFT}_{2}$ or higher dimensional CFT.

Finally, we have not evaluated all of the OTOC at order $\mathcal{O}\left(g^{4}\right)$, but we have calculated the contribution of the Schwarzian soft mode at order $\mathcal{O}\left(g^{4}\right)$. We have not considered the interaction of the matter fields which could also give a contribution to the OTOC. Unlike the soft mode, the contribution of matter fields might not be universal, but it would possibly depend on the details of models. Nevertheless, it would be interesting if the quantum correction to the chaos can constraint the matter interaction. 


\section{Acknowledgments}

We would like to thank Piljin Yi, Keun-Young Kim, Viktor Jahnke, Mitsuhiro Nishida, Martin Ammon and Robert de Mello Koch for extensive discussions. JY thanks the Erwin Schrodinger International Institute (ESI) where this work was initiated during the program "Higher Spins and Holography 2019". JY thank the Okinawa Institute of Science and Technology (OIST), the Gwangju Institute of Science and Technology (GIST) and the South China Normal University (SCNU) for the hospitality and generous support.

Open Access. This article is distributed under the terms of the Creative Commons Attribution License (CC-BY 4.0), which permits any use, distribution and reproduction in any medium, provided the original author(s) and source are credited.

\section{References}

[1] S.H. Shenker and D. Stanford, Black holes and the butterfly effect, JHEP 03 (2014) 067 [arXiv:1306.0622] [INSPIRE].

[2] S.H. Shenker and D. Stanford, Multiple shocks, JHEP 12 (2014) 046 [arXiv:1312.3296] [INSPIRE].

[3] D.A. Roberts, D. Stanford and L. Susskind, Localized shocks, JHEP 03 (2015) 051 [arXiv: 1409.8180] [INSPIRE].

[4] S.H. Shenker and D. Stanford, Stringy effects in scrambling, JHEP 05 (2015) 132 [arXiv: 1412.6087] [INSPIRE].

[5] J. Maldacena, S.H. Shenker and D. Stanford, A bound on chaos, JHEP 08 (2016) 106 [arXiv: 1503.01409] [INSPIRE].

[6] J. Yoon, A bound on chaos from stability, arXiv:1905.08815 [INSPIRE].

[7] S. Sachdev and J. Ye, Gapless spin fluid ground state in a random, quantum Heisenberg magnet, Phys. Rev. Lett. 70 (1993) 3339 [cond-mat/9212030] [INSPIRE].

[8] A. Kitaev, Hidden correlations in the Hawking radiation and thermal noise, KITP seminar February 12 (2015).

[9] A. Kitaev, A simple model of quantum holography, talks given at KITP, April 7 and May 27 (2015).

[10] J. Polchinski and V. Rosenhaus, The spectrum in the Sachdev-Ye-Kitaev model, JHEP 04 (2016) 001 [arXiv: 1601.06768] [INSPIRE].

[11] A. Jevicki, K. Suzuki and J. Yoon, Bi-local holography in the SYK model, JHEP 07 (2016) 007 [arXiv: 1603.06246] [INSPIRE].

[12] J. Maldacena and D. Stanford, Remarks on the Sachdev-Ye-Kitaev model, Phys. Rev. D 94 (2016) 106002 [arXiv: 1604.07818] [INSPIRE].

[13] A. Jevicki and K. Suzuki, Bi-local holography in the SYK model: perturbations, JHEP 11 (2016) 046 [arXiv : 1608.07567] [INSPIRE].

[14] D.J. Gross and V. Rosenhaus, A generalization of Sachdev-Ye-Kitaev, JHEP 02 (2017) 093 [arXiv: 1610.01569] [INSPIRE]. 
[15] W. Fu, D. Gaiotto, J. Maldacena and S. Sachdev, Supersymmetric Sachdev-Ye-Kitaev models, Phys. Rev. D 95 (2017) 026009 [arXiv:1610.08917] [inSPIRE].

[16] J. Yoon, SYK models and SYK-like tensor models with global symmetry, JHEP 10 (2017) 183 [arXiv: 1707.01740] [INSPIRE].

[17] J. Yoon, Supersymmetric SYK model: bi-local collective superfield/supermatrix formulation, JHEP 10 (2017) 172 [arXiv:1706.05914] [INSPIRE].

[18] P. Narayan and J. Yoon, Supersymmetric SYK Model with global symmetry, JHEP 08 (2018) 159 [arXiv: 1712 . 02647] [INSPIRE].

[19] F. Ferrari and F.I. Schaposnik Massolo, Phases of melonic quantum mechanics, Phys. Rev. D 100 (2019) 026007 [arXiv: 1903.06633] [INSPIRE].

[20] R. Gurau, The $1 / N$ expansion of colored tensor models, Annales Henri Poincaré 12 (2011) 829 [arXiv: 1011.2726] [INSPIRE].

[21] S. Carrozza and A. Tanasa, $O(N)$ random tensor models, Lett. Math. Phys. 106 (2016) 1531 [arXiv: 1512.06718] [INSPIRE].

[22] E. Witten, An SYK-like model without disorder, arXiv:1610.09758 [INSPIRE].

[23] R. Gurau, The complete $1 / N$ expansion of a SYK-like tensor model, Nucl. Phys. B 916 (2017) 386 [arXiv : 1611.04032] [INSPIRE].

[24] I.R. Klebanov and G. Tarnopolsky, Uncolored random tensors, melon diagrams and the Sachdev-Ye-Kitaev models, Phys. Rev. D 95 (2017) 046004 [arXiv:1611.08915] [InSPIRE].

[25] J. Maldacena, D. Stanford and Z. Yang, Conformal symmetry and its breaking in two dimensional Nearly Anti-de-Sitter space, PTEP 2016 (2016) 12C104 [arXiv:1606.01857] [INSPIRE].

[26] J. de Boer, E. Llabrés, J.F. Pedraza and D. Vegh, Chaotic strings in AdS/CFT, Phys. Rev. Lett. 120 (2018) 201604 [arXiv: 1709.01052] [INSPIRE].

[27] K. Murata, Fast scrambling in holographic Einstein-Podolsky-Rosen pair, JHEP 11 (2017) 049 [arXiv: 1708. 09493] [INSPIRE].

[28] A. Banerjee, A. Kundu and R.R. Poojary, Strings, branes, Schwarzian action and maximal chaos, arXiv: 1809.02090 [INSPIRE].

[29] A.L. Fitzpatrick and J. Kaplan, A quantum correction to chaos, JHEP 05 (2016) 070 [arXiv: 1601.06164] [INSPIRE].

[30] H. Chen, A.L. Fitzpatrick, J. Kaplan, D. Li and J. Wang, Degenerate operators and the 1/c expansion: Lorentzian resummations, high order computations and super-Virasoro blocks, JHEP 03 (2017) 167 [arXiv: 1606.02659] [INSPIRE].

[31] A.L. Fitzpatrick and J. Kaplan, On the late-time behavior of Virasoro blocks and a classification of semiclassical saddles, JHEP 04 (2017) 072 [arXiv: 1609.07153] [INSPIRE].

[32] E. Hijano, P. Kraus and R. Snively, Worldline approach to semi-classical conformal blocks, JHEP 07 (2015) 131 [arXiv: 1501.02260] [INSPIRE].

[33] E. Perlmutter, Virasoro conformal blocks in closed form, JHEP 08 (2015) 088 [arXiv: 1502.07742] [INSPIRE].

[34] Y. Hikida and T. Uetoko, Three point functions in higher spin $A d S_{3}$ holography with $1 / N$ corrections, Universe 3 (2017) 70 [arXiv:1708.02017] [INSPIRE]. 
[35] D. Stanford and E. Witten, Fermionic localization of the Schwarzian theory, JHEP 10 (2017) 008 [arXiv: 1703.04612] [INSPIRE].

[36] T.G. Mertens, G.J. Turiaci and H.L. Verlinde, Solving the Schwarzian via the conformal bootstrap, JHEP 08 (2017) 136 [arXiv:1705.08408] [INSPIRE].

[37] T.G. Mertens, The Schwarzian theory - Origins, JHEP 05 (2018) 036 [arXiv:1801.09605] [INSPIRE].

[38] Y.-H. Qi, Y. Seo, S.-J. Sin and G. Song, Correlation functions in Schwarzian liquid, Phys. Rev. D 99 (2019) 066001 [arXiv: 1804.06164] [INSPIRE].

[39] H.T. Lam, T.G. Mertens, G.J. Turiaci and H. Verlinde, Shockwave S-matrix from Schwarzian quantum mechanics, JHEP 11 (2018) 182 [arXiv:1804.09834] [INSPIRE].

[40] A. Blommaert, T.G. Mertens and H. Verschelde, The Schwarzian theory - A Wilson line perspective, JHEP 12 (2018) 022 [arXiv: 1806.07765] [INSPIRE].

[41] J. Cotler and K. Jensen, A theory of reparameterizations for AdS $S_{3}$ gravity, JHEP 02 (2019) 079 [arXiv: 1808.03263] [INSPIRE].

[42] D. Bagrets, A. Altland and A. Kamenev, Sachdev-Ye-Kitaev model as Liouville quantum mechanics, Nucl. Phys. B 911 (2016) 191 [arXiv:1607.00694] [INSPIRE].

[43] P. Saad, S.H. Shenker and D. Stanford, JT gravity as a matrix integral, arXiv:1903.11115 [INSPIRE].

[44] C. Peng, M. Spradlin and A. Volovich, Correlators in the $\mathcal{N}=2$ supersymmetric SYK model, JHEP 10 (2017) 202 [arXiv:1706.06078] [INSPIRE].

[45] A. Blommaert, T.G. Mertens and H. Verschelde, Fine structure of Jackiw-Teitelboim quantum gravity, JHEP 09 (2019) 066 [arXiv:1812.00918] [INSPIRE].

[46] T.G. Mertens and G.J. Turiaci, Defects in Jackiw-Teitelboim quantum gravity, JHEP 08 (2019) 127 [arXiv: 1904.05228] [INSPIRE].

[47] L.V. Iliesiu, S.S. Pufu, H. Verlinde and Y. Wang, An exact quantization of Jackiw-Teitelboim gravity, arXiv: 1905.02726 [INSPIRE].

[48] V. Jahnke, K.-Y. Kim and J. Yoon, On the chaos bound in rotating black holes, JHEP 05 (2019) 037 [arXiv: 1903.09086] [inSPIRE].

[49] P. Narayan and J. Yoon, Chaos in three-dimensional higher spin gravity, JHEP 07 (2019) 046 [arXiv: 1903.08761] [INSPIRE].

[50] M. Ammon, A. Castro and N. Iqbal, Wilson lines and entanglement entropy in higher spin gravity, JHEP 10 (2013) 110 [arXiv: 1306.4338] [INSPIRE].

[51] A. Castro, N. Iqbal and E. Llabrés, Wilson lines and Ishibashi states in $A d S_{3} / C F T_{2}, J H E P$ 09 (2018) 066 [arXiv: 1805.05398] [INSPIRE].

[52] R.R. Poojary, BTZ dynamics and chaos, arXiv:1812.10073 [INSPIRE].

[53] S. Grozdanov, K. Schalm and V. Scopelliti, Black hole scrambling from hydrodynamics, Phys. Rev. Lett. 120 (2018) 231601 [arXiv:1710.00921] [INSPIRE].

[54] M. Blake, H. Lee and H. Liu, A quantum hydrodynamical description for scrambling and many-body chaos, JHEP 10 (2018) 127 [arXiv:1801.00010] [INSPIRE].

[55] F.M. Haehl and M. Rozali, Effective field theory for chaotic CFTs, JHEP 10 (2018) 118 [arXiv: 1808.02898] [INSPIRE]. 
[56] M. Blake, R.A. Davison, S. Grozdanov and H. Liu, Many-body chaos and energy dynamics in holography, JHEP 10 (2018) 035 [arXiv:1809.01169] [INSPIRE].

[57] S. Grozdanov, On the connection between hydrodynamics and quantum chaos in holographic theories with stringy corrections, JHEP 01 (2019) 048 [arXiv:1811.09641] [INSPIRE].

[58] S. Grozdanov, P.K. Kovtun, A.O. Starinets and P. Tadić, The complex life of hydrodynamic modes, arXiv: 1904.12862 [INSPIRE].

[59] M. Blake, R.A. Davison and D. Vegh, Horizon constraints on holographic Green's functions, arXiv: 1904.12883 [INSPIRE]. 